\title{
A Study on Various Color Filter Array based Techniques
}

\author{
Simranpreet Kaur \\ Computer Science and Engineering \\ Global Institute Of Management and Emerging \\ Technologies, Amritsar
}

\begin{abstract}
A demosaicing algorithm is just a digital image process used to reconstruct full color image from the incomplete color samples output from a picture sensor overlaid with a color filter array (CFA). It is also known as CFA interpolation or color reconstruction. Most contemporary digital camera models acquire images using a single image sensor overlaid with a CFA, so demosaicing is the main processing pipeline required to render these images into a viewable format. Many modern digital camera models can save images in a natural format allowing the consumer to demosaicit using software, as opposed to utilizing the camera's built-in firmware. Thus demosaicing becomes and major area of research in vision processing applications. The key objective of the paper is to examine and analyze various image demosaicing techniques. The entire aim would be to explore various limitations of the earlier techniques. This paper ends up with the suitable gaps in earlier techniques.
\end{abstract}

\section{Keywords:-}

Demosaicing, Cfa, Bayer Layer, Smart Cameras.

\section{INTRODUCTION}

A demosaicing [1]- [4] is just a digital image process used to reconstruct full color image from incomplete color samples output from image sensor overlaid with a shade filter array (CFA). It's also referred to as CFA interpolation. Usage of digital cameras is spreading widely because they are easy image input devices. The increasing popularity of digital cameras has provided motivation to boost all elements of the digital photography signal chain. To lower cost, digital color cameras typically work with a single image detector. Color imaging with an individual detector requires the utilization of a Color Filter Array (CFA) which covers the detector array. In this arrangement each pixel in the detector samples the intensity of one of the many-color separations. The recovery of full-color images from a CFA-based detector requires a method of calculating values of another color separations at each pixel. These methods are commonly referred as color interpolation or color demosaicing algorithms. In a single-detector camera, varying intensities of light are measured at an rectangular grid of image sensors.

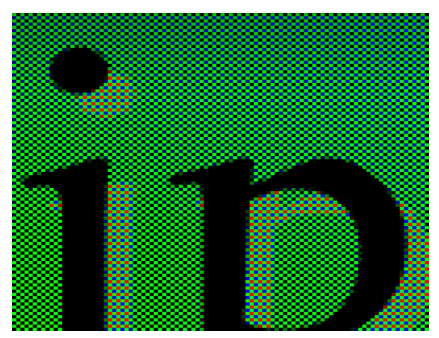

\author{
Richa Sharma \\ Assistant professor \\ Global Institute Of Management and Emerging \\ Technologies, Amritsar
}

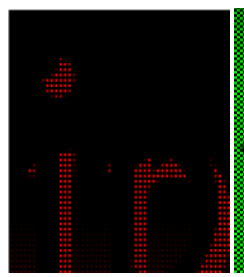

Red

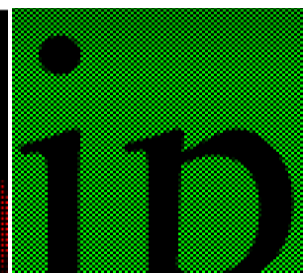

Green

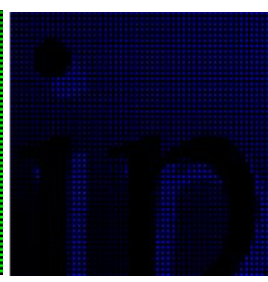

Blue
Figure 1: Bayer Filter Sa mple (adapted from [12])

A digital camera typically has means to reconstruct a whole RGB image using the above information. The resulting image could be something like this:

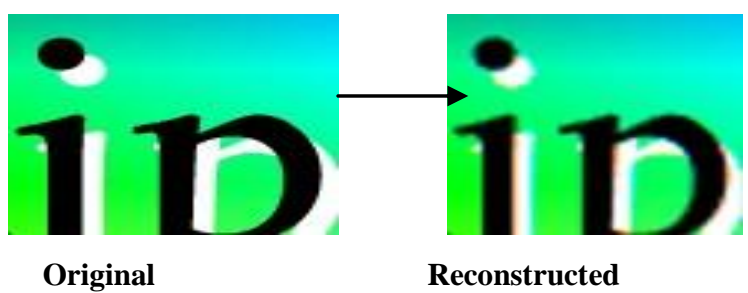

Figure 2: Reconstructed Image (adaptive from [12]

\section{COLOR FILTER ARRAY}

A color filter array is really a mosaic of color filters facing the image sensor. Commercially, the most commonly used CFA configuration is the Bayer filter illustrated here. This has alternating $\operatorname{red}(\mathrm{R})$ and green $(\mathrm{G})$ filters for odd rows and alternating green $(\mathrm{G})$ and blue (B) filters for even rows. There are twice as many green filters as red or blue ones, catering to the human eye's higher sensitivity to green light. Since along with sub sampling of a CFA by its nature results in aliasing, an optical anti-aliasing filter is typically put in the optical path involving the image sensor and the lens to reduce the false color artifacts (chromatic aliases) introduced by interpolation. Since each pixel of the sensor is behind a color filter, the output is a range of pixel values, each indicating a raw intensity of one of many three filter colors. Thus, an algorithm is required to estimate for every pixel along with levels for several color components, rather than a single component.

A Bayer filter mosaic is a color filter array (CFA) for arranging RGB color filters on a square grid of photo-sensors. Its particular arrangement of color filters is utilized in most single-chip digital image sensors utilized in digital camera models, camcorders, and scanners to make a color image. The filter pattern is $50 \%$ green, $25 \%$ red and $25 \%$ blue, hence can be called RGBG, GRGB or RGGB. The raw output of Bayerfilter cameras is referred to as a Bayer pattern image. Since each pixel is filtered to record only one of three colors, the info from each pixel cannot fully determine color on its own. To acquire a full-color image, various demosaicing algorithms can 
be utilized to interpolate a set of complete red, green, and blue values for each point

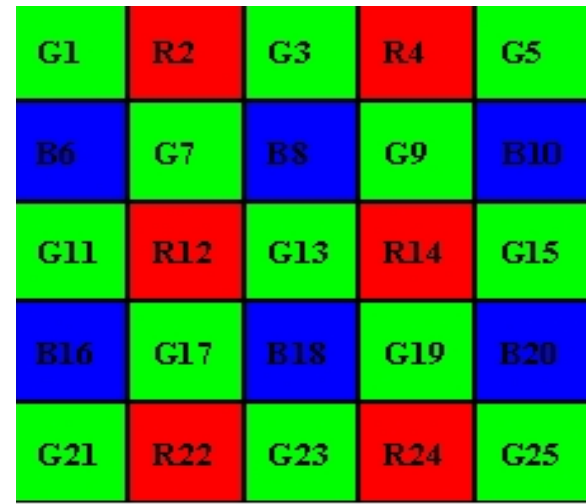

Figure 3: Bayer Pattern Array (adapted from [11])

During an image capture process, a digital camera performs various processing including auto-focus, white balance adjustment, color interpolation, color correction, compression and more. An important component of the imaging pipeline is color filter array (CFA) interpolation - i.e., to recover an fullresolution image from its CFA data.

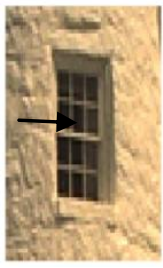

\begin{tabular}{|c|c|c|c|}
\hline$R$ & $G$ & $R$ & $G$ \\
\hline G & B & G & B \\
\hline R & G & R & G \\
\hline G & B & G & B \\
\hline
\end{tabular}

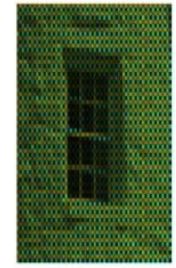

Original image CFA with Bayer pattern CFA image

Figure 4: CFA Image with Bayer Pattern (adapted from [13])

\section{COMMON DEMOSAICING ARTIFACTS}

Because sampling a scene employing a CCD with a Bayer pattern CFA measures only $33 \%$ of the info of the original scene, several artifacts occur consequently of demosaicing. Two of the most common are false coloring and zippering.

\section{False Color Artifact}

A consistent and unfortunate artifact of CFA demosaicing is what is recognized as false coloring. This artifact typically manifests itself along edges, where abrupt or unnatural shifts in color occur consequently of mis-interpolating across, rather than along, an edge. Figure 5 shows three images demosaiced with bilinear interpolation with types of false colors. Image (a) has an alternating pattern of red and blue highlights moving across the left edge of the windshield, along with some red and blue highlights on brighter portions of the mirror. Image (b) shows another view of the truck's windshield, where straight lines visible through the windshield appear as alternating red and yellow pixels. Image (c) shows false coloring amidst high frequency information in the Ford logo's lettering.

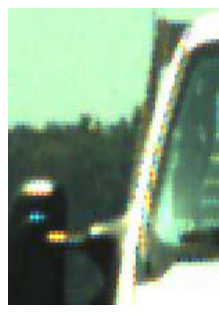

a)

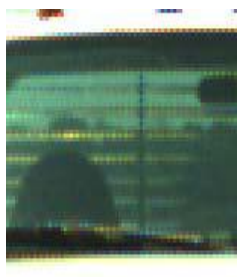

b)

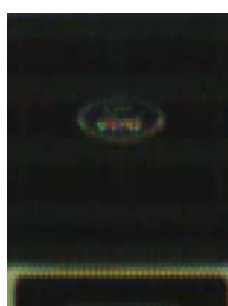

c)
Figure 5:- Three images depicting the false color demosaicing artifact. Image (a) shows the corner of a truck with false coloring on the side mirror and along the edge of the windshield. Image (b) shows the window of a truck with false coloring along the edges of the windshield and along edges showing through the windshield. Image (c) depicts the trucks insignia with false coloring amongst the high frequency information contained within (adaptive from

[11])

Zippering Artifact

Another side aftereffect of CFA demosaicing, which also occurs primarily along edges, is known as the zipper effect. Simply put, zippering is another name for edge blurring that happens in an on/off pattern along an edge. Figure 6 shows three images demosaicked with bilinear interpolation featuring the edge blurring zipper effect. Image (a) features a truck with zippering along the upper edge of the grill and also zippering along edges within the headlight. Image (b) features a person with zippering along the stripes in his shirt in addition to zippering along the fence poles in the background of the image. Image (c) shows a license plate with zippering along its six characters and more zippering along the upper edge of the bumper

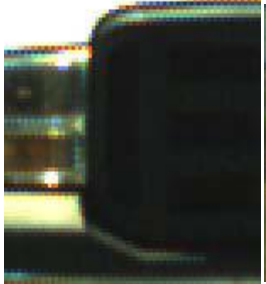

a)

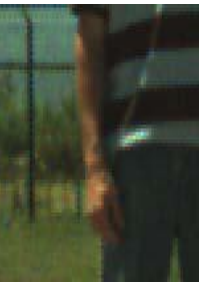

b)

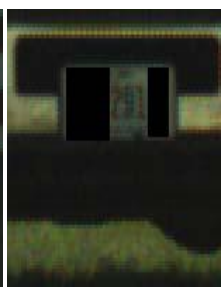

c)
Figure 6: Three images depicting the zippering artifact of CFA demosaicing. (a) features a truck with heavy zippering along edges of the grill and headlights. (b) features a person with zippering along his shirt's stripes and on the fence poles in the background. (c) features a license plate with zippering along its numbers as well as zippering along the edges of the bumper (adaptive from [11])

\section{LITERATURE SURVEY}

Lu Fang, Au.Oc and Katsaggelos A.k (2012) [9] presents an Adaptive Joint Demosaicing and Sub pixel-based Downsampling scheme (AJDSD) for single-sensor camera image, where the sub pixel-based down-sampling is adaptively and directly applied in Bayer domain, without the process of demosaicing. Simulation results demonstrate that when compared with conventional "demosaicing-first and down-sampling-later" methods, AJDSD achieves superior 
performance improvement in terms of computational complexity. As for visual quality, AJDSD is more effective in preserving high frequency details, resulting in much sharper and clearer results. Azgin H, Yaliman S and Hamzaoglu.I 2014 [2] presents that most cameras capture only 1 color channel per pixel utilizing a single image sensor. The images pass through a color filter array before being captured by the image sensor. Demosaicing is the method of reconstructing the missing color channels of the pixels in along with filtered image utilizing their available neighboring pixels. Alternating Projection (AP) is among the highest quality image demosaicing algorithms, and it's quite high computational complexity. Therefore, a good performance AP image demosaicing hardware is produced. Glotzbach et al, (2013) [10] stated that the green image is employed to incorporate high-frequency information and reduce aliasing in the red and blue images. First, the red and blue images are interpolated with a rectangular low pass filter based on the rectangular sampling grid. This fills in the missing values in the grid, but allows aliasing distortions to the red and blue output images. These output images will also be missing the highfrequency components needed to produce a sharp image. However, because the green image is sampled at a greater rate, the high-frequency information could be extracted from the green image to boost an initial interpolation of the red and blue images. An outside high pass filter and a vertical high pass filter are put on the green image. This allows the highfrequency information that the reduced sampling rate of the red and blue images cannot preserve. Maalouf et al. (2012) [14] has discussed a bandelet-based demosaicing method for color images and used a spatial mul-tiplexing type of color in order to obtain the luminance and the chrominance the different parts of the acquired image. Then, a luminance filter is employed to reconstruct the luminance component. Thereafter, based on the notion of maximal gradient of multivalued images, an extension of the bandelet representation for the case of multivalued images has proposed. Finally, demosaicing is completed by merging the luminance and all the chrominance components in the multivalued bandlet transform domain. Yung-Hsiang Chiu, Kuo-Liang Chung (2014) [5] has proposed a greater version of Yang et al.' s universal sub-sampling technique for compressing mosaic videos with arbitrary red-green-blue (RGB) color filter arrays in H.264/AVC. For the sub-sampled images with 4:2:0 format, Yang et al.' $s$ work retains the initial $\mathrm{Y}$ luma component, but samples the correct $\mathrm{U}$ and $\mathrm{V}$ chroma components according to the corresponding mosaic structure for better reconstructing R and B pixels. However, Yang et al.' $\mathrm{s}$ strategy is suffering from the RGB color deviations as a result of $U$ and $V$ chroma sub-sampling, which results in the quality degradation of the reconstructed mosaic videos. Chui and Chang proposed a story modification for the $\mathrm{Y}$ luma component as opposed to only retaining the Y luma component such that the RGB color deviation problem may be resolved. Chi-Yi Tsai and Kai-Tai Song (2007)[4] has reduced the aliasing error in red and blue channels by exploiting highfrequency information of the green channel. To make this happen, color-difference based edge-adaptive filtering and post-processing schemes are designed to reproduce along with values by exploiting the green channel information. For green channel interpolation, any of the existing image interpolation methods can be utilized and with the proposed algorithm. Moreover, a fresh adaptive interpolation method is presented for reconstructing the green channel from CFA samples. Jimmy Li et al. (2012) [7] has proposed an adaptive method in order to avoid inclusion of the bad pixels in the interpolation process has proposed. Only defective pixels which are considerably different from their surrounding neighbours is going to be deemed as bad and is going to be corrected. This is attained by adaptively varying the order of interpolation to ensure that along interpolation is shorter in case a bad pixel is found nearer to the pixel being interpolated. The bad pixels are located with a median-based multi-shell filter structure. Sevinc Bayram and Husrev T. Sencar (2008) [3] employ two methods and define some image characteristics which are utilized as features in designing classifiers that distinguish between digital camera models. The very first method tries to estimate demosaicing parameters assuming linear model while the second one extracts periodicity features to detect simple forms of demosaicing. To find out the reliability of the designated image features in differentiating the foundation camera model, we consider both images taken under similar settings at fixed sceneries and images taken under independent conditions. O. Losson and A.Porebski (2013) [13] has produced a shade image by which the 2 missing color components need to be estimated at each pixel of the corresponding CFA image. This process is commonly referred to as demosaicing, and its result because the demosaiced color image. Since demosaicing methods want to produce "perceptually satisfying"demosaiced color images, they attempt in order to avoid color artifacts. Because this is often attained by filtering, demosaicing schemes tend to alter the local texture information that is, however, useful to discriminate texture images. To avoid this dilemma while exploiting color information for texture classification, it might be relevant to compute texture descriptors directly from CFA images. From chromatic co-occurrence matrices (CCMs) that capture the spatial interaction between color components, we derive new descriptors (CFA CCMs) for CFA texture images. Color textures are then com- pared in the form of the similarity between their CFA CCM. Jayanta Mukherjee and Manfred K. Lang (2005) [16] present methods for interpolating the CFA data in the YUV color space to acquire the full color image. The resulting color images could possibly be directly utilized in the DCT based JPEG compression scheme. Mukherje and K.Lang also used the thought of sub-band DCT computation for interpolating the average person components of color images in the DCT domain. Centered on a simple strategy for computing the $\mathrm{Y}, \mathrm{U}$, and $\mathrm{V}$ components, several modifications are also proposed for improving the grade of the reconstructed images. We've observed that median filtering of the chrominance components improves the visual quality of the interpolated color image. .Pekkucuksen et al. (2012) [17] has presented a simple edge strength filter to interpolate the missing color values adaptively. Whilst the filter is readily applicable to the Bayer mosaic pattern, we argue that the exact same idea could possibly be extended to other mosaic patterns and describe its application to the Lukac mosaic pattern. Clearly outperforms other available solutions with regards to CPSNR. We believe that edge oriented, directional approach could show to be helpful for other CFA patterns as well. WenJan Chen and Pei-Yu Chang (2011) [12] has proposed a demosaicing method to prevent the occurrence of color artifacts. By detecting the edge characteristics of a digital image, accurate weights could be obtained for image 
interpolation, before refinement is made in post-processing. Demosaicing is the first faltering step of image processing of digital still cameras and has been built-into the look of many different digital still cameras. If noise and blurred edges exist from the onset of image reconstruction, a post-processing can perform little to boost the grade of the reconstructed image. Chung et al. (2012) [13] has presented a highly effective decision-based demosaicing algorithm for Bayer images. An enhanced edge-sensing measure called enhanced integrated gradient can support more gradient information from various color intensity and color difference planes under the directional compatibility constraint. An adaptive green plane enhancement which works together the enhanced integrated gradient can also be proposed to help expand increase the efficiency of the algorithm. Gang and Chang (2012) [11] has presented an adaptive demosaicing algorithm by exploiting the non-local similarity and the area correlation in the color filter array image. First, the absolute most flattest non-local image patches are searched in the searching window devoted to the estimated pixel. Second, the patch, that will be the absolute most like the current patch, is selected among the absolute smoothest nonlocal patches. Third, in line with the similar degree and the area correlation degree, the obtained non local image patch and the current patch are adaptively chosen to estimate the missing color samples. Ling Shao and Amin Ur Rehman (2014) [18] Most cameras of today use a single CCD image sensor with alternating arrangement of red, blue and green colour filters in what is called a Bayer pattern. To extract a full colour image, a demosaicing strategy needs to be applied. In this paper we propose a content adaptive demosaicing strategy utilising structure analysis and correlation between the red, green and blue planes. Those two aspects are used for the classification of a block of pixels to generated trained filters. The proposed method aims to reconstruct a superior quality demosaiced image from a Bayer pattern in a colour filter array efficiently. Experimental results show that the proposed strategy performs comparatively as higher priced methods. Fang et al. (2012) [16] has proposed a fast frequency-domain analysis approach for joint demosaicing and sub-pixel based down-sampling of single sensor Bayer images. In this, we integrate demosaicing into down-sampling by directly performing sub-pixel based down sampling in the bayer domain, due to that your computational complexity is reduced. Dohyoung Lee and Konstantin N. Plataniotis (2012) [17] has proposed pipeline which contains some pre-processing operations followed with a JPEG XR encoding module. A deinterleaving step separates the CFA image to sub-images of just one color channel, and each sub-image is processed with a proposed weighted template matching prediction. The utilized JPEG XR codec allows the compression of HDR data at low computational cost. Maschal and Susan Young (2012) [18] has proposed two new no-reference quality assessment algorithms. These algorithms provide a relative comparison of two demosaicing algorithms by measuring the clear presence of two common artifacts, zippering and false coloring, in their output images. The first algorithm, the edge slope measure, tests the overall sharpness of all the three color channels, thus estimating the relative edge reconstruction accuracy of every demosaicing algorithm. The next algorithm, the false color measure, estimates deviations from the established constant color difference image model and performs on green-red and green blue color difference planes, therefore estimating the red and blue channel reconstruction of every demosaicing algorithm. We evaluate and rank common demosaicing algorithms using these new algorithms. Yu Zhang and Guangyi Wanga (2011) [11] has proposed the wavelet sub-band decomposition and synthesis are applied to interpolate the CFA data with signal-dependent noise model. The major contributions of the work include: (1) The combination of LMMSE and statistical calculation in wavelet domain are utilized to suppress the signal-dependent noise, which will be separated into additive noise and multiplicative noise. (2) In CFA data, it has been verified that the quantitative relationship between the existing pixel and the adjacent pixel, which locate in the exact same edge. Brice Chaix de Lavarene and David Alleyson (2006) has proposed a modification of the stacked notation of super pixels, which allows a highly effective computing of the LMMSE solution from a graphic database. Moreover, this formalism is used to decompose the CFA sampling right into a sum of a luminance estimator and a chrominance projector. This decomposition allows interpreting estimated filters in term of these spatial and chromatic properties and results in a solution with lower computational complexity than other LMMSE approaches for the exact same quality. Barbara Daja and Mirjana Bonkovic (2013) [8] show that correct modeling of the Bayer pattern in the generative process improves the super resolution performance for colour images , and an algorithm that incorporates the 2 colour prior into the probabilistic model is designed. The algorithm presented in this paper centers on the classes of images which have two dominant colours, i.e. all of the areas in the image are uniformly coloured. Dongjae Leeand Byung-Joon Baekone (2012) [7] has proposed two-layer color filter array, that includes a full resolution in $\mathrm{G}$ channel and a half resolution in $\mathrm{R} / \mathrm{B}$ channel. To evaluate the performance, simple demosaic algorithms are presented. A two-layer color filter array to acquire a high quality image and demosaic algorithms to interpolate the proposed two-layer color filter array. Although demosaic and color filter array design methods have been improved together, recent research results show the performance saturation since the sub-sampling of color channels is inevitable. To overcome this limitation, multilayer color filter arrays have been developed to acquire two or three color data at single pixel position. Sebastien Courroux and Stephane Chevobbe (2013) [6] have investigated the chance to utilize the wavelet representation to perform high quality image processing algorithms at a lowered computational complexity than utilising the spatial representation. To reproduce such conditions, demosaicing, denoising, contrast correction and classification algorithms are executed over several popular embedded cores (Leon3, Cortex A9 and DSP C6x). Waveletbased image reconstruction shows higher image quality and lower computational complexity $(3 \mathrm{x})$ than usual spatial reconstruction. 
Table .1: Comparison Table

\begin{tabular}{|c|c|c|c|c|c|c|}
\hline $\begin{array}{l}\text { REF } \\
\text { NO. }\end{array}$ & $\begin{array}{c}\text { AUTHOR } \\
\text {-NAME }\end{array}$ & YEAR & TECHNIQUE & FEATURES & LIMITATIONS & $\begin{array}{c}\text { POST- } \\
\text { PROCESSING }\end{array}$ \\
\hline 1. & $\begin{array}{c}\text { Brice } \\
\text { Chaix de } \\
\text { Lavarane }\end{array}$ & 2007 & $\begin{array}{l}\text { LMME } \\
\text { (Linear } \\
\text { minimum } \\
\text { mean square } \\
\text { error) }\end{array}$ & $\begin{array}{l}\text { i)Good trade- off between quality } \\
\text { and computational cost for } \\
\text { embedded system. } \\
\text { ii) High efficiency }\end{array}$ & $\begin{array}{l}\text { i)Directional interpolation } \\
\text { results in reconstruction } \\
\text { with less artifacts. }\end{array}$ & Yes \\
\hline 2. & $\begin{array}{c}\text { Barbara } \\
\text { Daja }\end{array}$ & 2013 & $\begin{array}{c}\text { Super } \\
\text { resolution } \\
\text { demosaicing }\end{array}$ & $\begin{array}{l}\text { i) Improves super resolution } \\
\text { performance for color images. } \\
\text { ii) Far better results than MAP } \\
\text { approach. }\end{array}$ & $\begin{array}{l}\text { i)Is not useful when three } \\
\text { or more colored areas } \\
\text { meet in a single point or } \\
\text { shaded or textured areas. }\end{array}$ & Yes \\
\hline 3. & $\begin{array}{c}\text { Sevinc } \\
\text { Bayram }\end{array}$ & 2008 & $\begin{array}{l}\text { Linear model } \\
\quad \text { for } \\
\text { differentiating } \\
\text { digital camera } \\
\text { models. }\end{array}$ & $\begin{array}{l}\text { i)Determine the camera model } \\
\text { which is used to capture image. } \\
\text { ii)Decide particular model of } \\
\text { camera among large number of } \\
\text { camera models. } \\
\text { iii)More reliably identify } \\
\text { individual camera. }\end{array}$ & $\begin{array}{l}\text { Robustness of technique } \\
\text { to malicious processing. }\end{array}$ & No \\
\hline 4. & $\begin{array}{l}\text { Wen-Jan- } \\
\text { Chen }\end{array}$ & 2011 & $\begin{array}{l}\text { Demosaicing } \\
\text { algorithm } \\
\text { based on edge } \\
\text { property for } \\
\text { CFA }\end{array}$ & $\begin{array}{l}\text { i) Prevent occurrence of color } \\
\text { artifacts. } \\
\text { ii)Enhance image qualiy. } \\
\text { iii)Give high PSNR value }\end{array}$ & $\begin{array}{l}\text { i)Interpolation of only } \\
\text { green - pixel values. }\end{array}$ & No \\
\hline 5. & $\begin{array}{l}\text { Yung- } \\
\text { Hsiang- } \\
\text { Chiu }\end{array}$ & 2014 & $\begin{array}{c}\text { Universal } \\
\text { sub-sampling } \\
\text { strategy }\end{array}$ & $\begin{array}{l}\text { i)Better quality of mosaic and } \\
\text { full-color videos. }\end{array}$ & $\begin{array}{l}\text { i)Not valid for digital time } \\
\text { delay integration images } \\
\text { in which each pixel has } \\
\text { two RGB primary color } \\
\text { components. }\end{array}$ & Yes \\
\hline 6. & Ling Shao & 2014 & $\begin{array}{l}\text { ADRC } \\
\text { (Adaptive } \\
\text { dynamic } \\
\text { range coding) } \\
\text { strategy }\end{array}$ & $\begin{array}{l}\text { i)Less expensive. } \\
\text { ii)Less complex in multi-order } \\
\text { differential operations. } \\
\text { iii)Directional interpolation is } \\
\text { performed to evaluate missing } \\
\text { pixels. }\end{array}$ & $\begin{array}{l}\text { i)Cost to maintain } \\
\text { memory requirements is } \\
\text { higher. }\end{array}$ & Yes \\
\hline 7. & $\begin{array}{l}\text { Sebastein } \\
\text { Courroux }\end{array}$ & 2010 & $\begin{array}{l}\text { Wavelet } \\
\text { Decomposit- } \\
\text { ion }\end{array}$ & $\begin{array}{l}\text { i)Reduced computational } \\
\text { complexity. } \\
\text { ii)Low memory requirements. } \\
\text { iii)Increase recognition rates of } \\
\text { face. }\end{array}$ & $\begin{array}{l}\text { i)High cost of wavelet } \\
\text { transform. } \\
\text { ii)Very time consuming to } \\
\text { meet real-time } \\
\text { requirements }\end{array}$ & Yes \\
\hline 8. & $\begin{array}{l}\text { Chi-Yi- } \\
\text { Tsai }\end{array}$ & 2006 & $\begin{array}{l}\text { EADA } \\
\text { (Edge } \\
\text { adaptive } \\
\text { demosaicing } \\
\text { algorithm) }\end{array}$ & $\begin{array}{l}\text { i)Reduced color artifacts. } \\
\text { ii)Reduced aliasing error in red- } \\
\text { blue channels. } \\
\text { iii)Better PSNR and CIELAB } \\
\text { measures. }\end{array}$ & $\begin{array}{l}\text { i)Cannot reconstruct green } \\
\text { channel with minimum } \\
\text { interpolation error. }\end{array}$ & No \\
\hline 9. & $\begin{array}{l}\text { Dohyoung } \\
\text { Lee }\end{array}$ & 2012 & $\begin{array}{l}\text { Lossless CFA } \\
\text { image } \\
\text { compression }\end{array}$ & $\begin{array}{l}\text { i)Compression of HDR data at } \\
\text { low computational cost. } \\
\text { ii)Higher efficiency. }\end{array}$ & $\begin{array}{l}\text { i)Blurrness of edges are } \\
\text { observed. }\end{array}$ & No \\
\hline
\end{tabular}




\begin{tabular}{|c|c|c|c|c|c|c|}
\hline 10. & O.Losson & 2013 & $\begin{array}{l}\text { CFA } \\
\text { Chromatic } \\
\text { Co- } \\
\text { Occurrence } \\
\text { Matrices } \\
\text { (CCM's) }\end{array}$ & $\begin{array}{l}\text { i)Efficient in texture classification } \\
\text { ii)CFA CCM's are derived for } \\
\text { CFA texture images. } \\
\text { iii)Less time consuming than } \\
\text { CCM's. }\end{array}$ & $\begin{array}{l}\text { i)Cannot classify texture } \\
\text { when acquired by a single } \\
\text { sensor color camera. }\end{array}$ & Yes \\
\hline 11. & $\begin{array}{l}\text { Jayanta } \\
\text { MUkherje } \\
\text { e }\end{array}$ & 2005 & $\begin{array}{c}\text { MRF } \\
\text { (Markov } \\
\text { random field) } \\
\text { Processing }\end{array}$ & $\begin{array}{l}\text { i)Edges are enhanced. } \\
\text { ii)Smooth the interpolated } \\
\text { images. } \\
\text { iii)PSNR is improved. }\end{array}$ & $\begin{array}{l}\text { i)Poor recovery of edges } \\
\text { of images. }\end{array}$ & Yes. \\
\hline 12. & $\begin{array}{l}\text { Hasan } \\
\text { Azgin }\end{array}$ & 2014 & $\begin{array}{l}\text { Alternating } \\
\text { projection } \\
\text { (AP) } \\
\text { algorithm }\end{array}$ & $\begin{array}{l}\text { i)A high performance AP image } \\
\text { demisaicing hardware is } \\
\text { proposed. }\end{array}$ & $\begin{array}{l}\text { i)Lower computational } \\
\text { speed. }\end{array}$ & No \\
\hline 13. & $\begin{array}{c}\text { Aldo } \\
\text { Maalouf }\end{array}$ & 2012 & $\begin{array}{c}\text { Spatial } \\
\text { multiprocessi } \\
\text { ng model }\end{array}$ & $\begin{array}{l}\text { i)Bandlet based demosaicing } \\
\text { method is proposed. } \\
\text { ii)Reconstruction of luminance } \\
\text { component. } \\
\text { iii)Chrominance components are } \\
\text { obtained. }\end{array}$ & $\begin{array}{l}\text { i)Computational cost is } \\
\text { higher. }\end{array}$ & Yes \\
\hline 14. & $\begin{array}{l}\text { Pekkucuk } \\
\text { sen }\end{array}$ & 2012 & $\begin{array}{l}\text { Edge strength } \\
\text { filter }\end{array}$ & i)Interpolation of missing values. & $\begin{array}{l}\text { i)Edge-oriented } \\
\text { directional approach is not } \\
\text { applicable. }\end{array}$ & Yes \\
\hline 15. & $\begin{array}{l}\text { Robert } \\
\text { A.MAsch } \\
\quad \text { al }\end{array}$ & 2011 & $\begin{array}{l}\text { Linear and } \\
\text { cubic } \\
\text { interpolation }\end{array}$ & $\begin{array}{l}\text { i)Edge slope measures. } \\
\text { ii)False color measures. }\end{array}$ & $\begin{array}{l}\text { i)Not much improvement } \\
\text { in visual quality. }\end{array}$ & No \\
\hline 16. & Yu-Zhang & 2011 & $\begin{array}{l}\text { Wavelet sub- } \\
\text { band } \\
\text { decompositio } \\
n\end{array}$ & $\begin{array}{l}\text { i)Suppress the signal dependent } \\
\text { noise. } \\
\text { ii)Less computational cost. } \\
\text { iii)Preserve image details. }\end{array}$ & $\begin{array}{l}\text { i)Cannot determine } \\
\text { relationship between } \\
\text { current and adjacent pixel } \\
\text { values. } \\
\text { ii)Less accurate color } \\
\text { interpolation }\end{array}$ & Yes \\
\hline
\end{tabular}

\section{GAPS IN LITERATURE}

1. The effectation of the demosaicing artifacts due to color channel interpolation has already been neglected in the prevailing research.

2. The utilization of the soft computing to efficiently interpolate the total color image has already been ignored.

\section{CONCLUSION}

A shade filter array is just a mosaic of color filters facing the image sensor. Commercially, the absolute most commonly used CFA configuration is the Bayer filter illustrated here. By conducting the review it's been discovered that the most of the existing literature has neglected many issues. The effectation of the demosaicing artefacts because of color channel interpolation has also been neglected in the existing research. The utilization of the soft computing to efficiently interpolate the total color image has also been ignored. In forseeable future we will improve this content based color filter array further by utilizing Ant colony algorithm based interpolation is likely to be used. It will reduce the situation of color artefacts by using the color channel normalization.

\section{REFERENCES}

[1] Alvarez, Luis, Pierre-Louis Lions, and Jean-Michel Morel. "Image selective smoothing and edge detection by nonlinear diffusion. II." SIAM Journal on numerical analysis 29, no. 3 (1992): 845-866.

[2] Azgin, Hasan, Serkan Yaliman, and Ilker Hamzaoglu.2014"A high performance alternating projections image demosaicing hardware." In Field Programmable Logic and Applications (FPL), 2014 24th International Conference on, pp. 1-4. IEEE.

[3] Bayram, Sevinc, Husrev T. Sencar, and Nasir Memon 2008 "Classification of digital camera-models based on demosaicing artifacts." digital investigation 5, no. 1 : 4959.

[4] Chen, Wen-Jan, and Pei-Yu Chang 2012 "Effective demosaicking algorithm based on edge property for color 
filter arrays." Digital Signal Processing 22, no. 1 : 163169.

[5] Chiu, Yung-Hsiang, Kuo-Liang Chung, and Chien-Hsiung Lin 2014 "An improved universal subsampling strategy for compressing mosaic videos with arbitrary RGB color filter arrays in H. 264/AVC." Journal of Visual Communication and Image Representation 25, no. 7 : 1791-1799.

[6] Courroux, Sébastien, Stéphane Chevobbe, Mehdi Darouich, and Michel Paindavoine 2013 "Use of wavelet for image processing in smart cameras with low hardware resources." Journal of Systems Architecture 59, no. 10 : 826-832.

[7] Criminisi, Antonio, Patrick Pérez, and Kentaro Toyama. 2004"Region filling and object removal by exemplarbased image inpainting." Image Processing, IEEE Transactions on 13, no. $9: 1200-1212$.

[8] Džaja, Barbara, Mirjana Bonković, and Ljubomir Malešević 2013"Solving a two-colour problem by applying probabilistic approach to a full-colour multiframe image super-resolution." Signal Processing: Image Communication 28, no. 5 : 509-521.

[9] Fang, Lu, Oscar C. Au, Yan Chen, Aggelos K. Katsaggelos, Hanli Wang, and Xing Wen 2012 "Joint Demosaicing and Subpixel-Based Down-Sampling for Bayer Images: A Fast Frequency-Domain Analysis Approach." Multimedia, IEEE Transactions on 14, no. 4 : 1359-1369.

[10] Glotzbach, John W., Ronald W. Schafer, and Klaus Illgner 2001"A method of color filter array interpolation with alias cancellation properties." In Image Processing, 2001. Proceedings. 2001 International Conference on, vol. 1, pp. 141-144. IEEE.

[11] Guo-gang, Wang, Zhu Xiu-chang, and Gan Zong-liang 2012 "Image demosaicing by non-local similarity and local correlation." In Signal Processing (ICSP), 2012 IEEE 11th International Conference on, vol. 2, pp. 806810. IEEE.
[12] Lee, Dohyoung, and Konstantinos N. Platanioti” 2012 "Lossless compression of HDR color filter array image for the digital camera pipeline." Signal Processing: Image Communication 27, no. $6: 637-649$.

[13] Losson, Olivier, Alice Porebski, Nicolas Vandenbroucke, and Ludovic Macaire 2013 "Color texture analysis using CFA chromatic co-occurrence matrices."Computer Vision and Image Understanding 117, no. $7:$ 747-763.

[14] Maalouf, Aldo, M-C. Larabi, and S. Susstrunk 2012"An efficient demosaicing technique using geometrical information." In Image Processing (ICIP), 2012 19th IEEE International Conference on, pp. 2789-2792. IEEE.

[15] Maschal, Robert A., S. Susan Young, Joseph P. Reynolds, Keith Krapels, Jonathan Fanning, and Ted Corbin 2013"New image quality assessment algorithms for CFA demosaicing." Sensors Journal, IEEE 13, no. 1 : 371-378.

[16] Mukherjee, Jayanta, Manfred K. Lang, and Sanjit K. Mitra 2005 "Demosaicing of images obtained from single-chip imaging sensors in YUV color space." Pattern recognition letters 26, no. 7 : 985-997.

[17] Pekkucuksen, Ibrahim, and Yucel Altunbasa 2011. "Edge oriented directional Color Filter Array interpolation." In Acoustics, Speech and Signal Processing (ICASSP), 2011 IEEE International Conference on, pp. 993-996. IEEE.

[18] Shao, Ling, and Amin Ur Rehman 2014 "Image demosaicing using content and colour-correlation analysis." Signal Processing 103 : 84-91.

[19] Tang, Ketan, Oscar C. Au, Lu Fang, Yuanfang Guo, and Jiahao Pang 2013 "Chroma Replacing and adaptive Chroma Blending for subpixel-based downsampling." InMultimedia Signal Processing (MMSP), 2013 IEEE 15th International Workshop on, pp. 212-217. IEEE.

[20] Tsai, Chi-Yi, and Kai-Tai Song 2013"A new edgeadaptive demosaicing algorithm for color filter arrays." Image and Vision Computing 25, no. 9: 14951508. 